\title{
Where and how to find bio-inspiration?
}

\section{A comparison of search approaches for Bio-Inspired Design}

\author{
Mart Willocx $^{\mathrm{a} *}$, Amir Ayali $^{\mathrm{b}}$, Joost R. Duflou ${ }^{\mathrm{a}, \mathrm{c}}$ \\ a KU Leuven, Department of Mechanical Engineering, Celestijnenlaan 300A, box 2422, 3001, Leuven, Belgium \\ b School of Zoology \& Sagol school of Neuroscience, Tel Aviv University, Tel Aviv 6997801, Israel \\ $c$ Member of Flanders Make \\ * Corresponding author: mart.willocx@kuleuven.be
}

\section{Abstract}

Biologicalisation calls for the integration of biological knowledge in manufacturing. Although biologists have been cataloguing biological knowledge for centuries, for the non-biologist finding this inspiration as input for bio-inspired design is a major challenge. In this paper, three different methods are used to find bio-inspiration for a case study on the clamping interface of a flexible mobile machining unit: the AskNature database, a natural language processing (NLP) approach, drawing on a large corpus of biological publications, and an informal consultation of an expert biologist as could be organised by a design office. The comparison of the retrieved principles with AskNature as a baseline indicates that the NLP approach allows retrieving publications about relevant biological strategies with a good recall performance, without involving an expert. However, extracting the working principles from the biological articles retrieved with the NLP approach is found to be error-prone and time consuming.

\section{Keywords \\ - Biologicalisation \\ - Biologically inspired design \\ - Design method \\ - Creativity and ideation \\ - Knowledge management}

\section{Introduction}

For thousands of years, nature has been an inspiration source for designers and engineers. From Leonardo da Vinci's flying machines, inspired by birds, to the invention of Velcro, using the knowledge captured in nature has yielded effective designs (Vincent et al. 2006). More recently, the biological transformation in manufacturing aims to use bio-inspired principles, functions and structures for developing more sustainable manufacturing systems, relying on the analogical transfer of the underlying principle from biology to engineering to create innovative ideas (Hashemi Farzaneh et al. 2019; Byrne et al. 2018). Various studies have validated the potential of using biological inspiration during the conceptual design phase to generate out-of-the-box solutions. (Keshwani et al. 2017) and (Vandevenne et al. 2016) observed an increase in the novelty of the generated concepts when compared to brainstorming and (Ahmed-Kristensen et al. 2014) also found an increase in novelty when compared to random images as an inspiration source.

Despite these promising results, only a limited number of bio-inspired products are commercially available (Wanieck et al. 2017). Many of the existing bio-inspired designs were created by chance 
observation of a relevant biological phenomenon (Nagel 2010). Most engineers do not possess biological background knowledge and have a hard time identifying, filtering and understanding relevant biological strategies to apply to their design problem, turning the search for relevant biological strategies into a frustrating and time-consuming task (Fayemi et al. 2017; Graeff et al. 2019; Kruiper et al. 2018).

In order to introduce a more systematic method, a range of systematic bio-inspired design processes have been proposed (Lenau et al. 2018). Vandevenne et al. summarised the different phases in this process as problem formulation, search for relevant biological strategies, selection and analysis of these strategies and finally the knowledge transfer to the engineering domain (Vandevenne et al. 2015). Figure 1 provides a schematic overview of this process. During the search phase, relevant biological strategies are retrieved, from which the interesting principles are extracted during the analysis phase. Finally, these biological principles serve as inspiration in the engineering domain during the knowledge transfer phase.

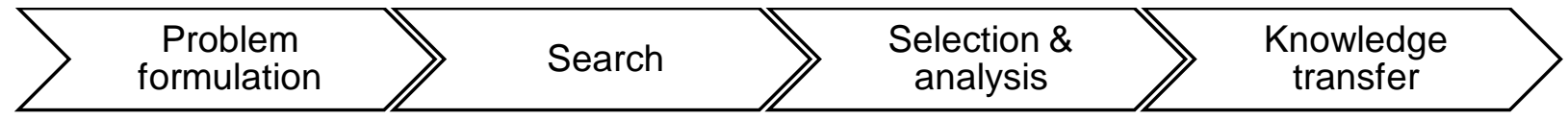

Figure 1: The phases of the systematic bio-inspired design process as identified by Vandevenne et al. (2015).

Researchers have developed a number of methods and supporting tools for each phase of the process. Here, the focus is on identifying relevant biological strategies for a given engineering problem: the search phase. The proposed approaches for this phase take three different forms: consulting a specialist directly by including a biologist in the search procedure, using biological strategies stored in a specially prepared database, and the direct use of biological literature as a natural language source of knowledge.

This paper compares the different approaches for retrieving biological inspiration through observations made in the context of a specific case study on the attachment mechanism of a mobile machining unit, as described in (van Houten et al. 2020). The first sections introduce the different approaches for retrieving bio-inspiration and the design requirements from the use case. Next, the concrete methods applied here are introduced and, if necessary, the design requirements are converted to the required search input. Finally, the retrieved biological strategies are analysed and the results of the different methods are compared and discussed.

\section{Retrieving bio-inspiration}

When collaborating with a biologist, a trained domain expert performs the search for biological inspiration, complementing the lack of biological knowledge of the engineer. In addition, Lenau et al. note that the biologist can do a pre-evaluation of the usefulness of the biological strategy, reducing time spent on trying to understand irrelevant material (Lenau et al. 2011). Schöfer et al. further identify that disciplinary diversity in a design team promotes an in-depth problem formulation and solution proposition (Schöfer et al. 2018). Despite these advantages, Graeff et al. noticed that only a very limited number of the proposed approaches aim to include a biologist in the design process (Graeff et al. 2019). Some experiments have been conducted with a biologist in the design team to aid the identification of bio-inspiration: (Helms et al. 2009) reports on a study with 45 students, of which 6 were biologists, (Helten et al. 2011) and (Lenau et al. 2011) report on student teams with one biologist and respectively 
two and a single engineering student. In these studies, the biologist relies on his/her previous knowledge to find relevant biological strategies, without using a systematic search method.

Shu et al. warn that a biologist might be biased towards his/her own area of expertise and fail to present an objective variety of relevant biological information (Shu et al. 2011). In addition, Graeff et al. note that in order to be able to recall a representative set of strategies and principles, the biologist will need to possess a very broad knowledge of nature. This is in direct contrast with most biologists, who are doing in-depth research on a very specific subset of biology (Graeff et al. 2019). Furthermore, due to the difference in background, misunderstandings between the engineers and biologists are common (Helten et al. 2011). Sketches and drawings were explored as communication aids between both domains (Hashemi Farzaneh et al. 2015).

In an attempt to bypass the background knowledge requirement and bridge the search gap between biology and engineering, researchers developed a number of specialized knowledge repositories aimed at supporting design and engineering activities. Here, experts convert the biological strategies to a format that is easily searchable for engineers. For instance, the AskNature taxonomy allows searching for a relevant strategy by locating the desired function in a list of categories. In addition, the biologists cataloguing the strategies create a short summary, aiding the next phases of the design process (Deldin et al. 2014). The enormous effort required for the creation of such databases is their biggest drawback. Graeff notes that even though AskNature is the biggest database for bio-inspired design - containing 1724 strategies (Biomimicry Institute 2020) - it still is far from being sufficiently populated to cover all potentially relevant organisms in an exhaustive way (Graeff et al. 2019).

The natural language processing (NLP) based approach leverages the knowledge captured in biological literature, bypassing the scalability issues presented by having to manually build and maintain a database. Shu et al. developed a tailored search for extracting biological phenomena from biology textbooks, starting from a functional keyword search (Shu 2010). Vandevenne et al. leveraged dimensional reduction to allow scalable search in a large corpus of biological articles (Vandevenne et al. 2015). This search method returns a ranked list of relevant biological articles, from which the end-user has to manually extract the underlying principles, thus only supporting the search phase.

\section{Design requirements mobile machining unit}

Traditionally a workpiece that has to be machined is mounted on a larger machining station. When machining is necessary on a large component, for example a wind turbine blade, this method requires an even larger machining base. A mobile machining unit that clamps to the workpiece and can easily be repositioned could replace these large machines. Full details of the development of the clamping interface and the design requirements are presented in (van Houten et al. 2020). Here, the focus is on the search for the bio-inspiration required to develop the clamping interface between mobile machining tool and workpiece.

The design requirements steer the development of a clamping interface that is strong enough to absorb machining forces and that is easily reversible to allow the envisaged machine to move around on the workpiece autonomously. The clamping conditions are defined as tilted, vertical and upside down orientations on a range of rough and smooth surfaces, mainly steel, aluminium and composites. No adaptations can be made to the surface to facilitate the clamping. Furthermore, the attachment mechanism should not cause damage to the surface. Figure 2 schematically indicates the different 
orientations on a wind turbine blade and gives an indication of the size of a common wind turbine blade (Wang et al. 2015).

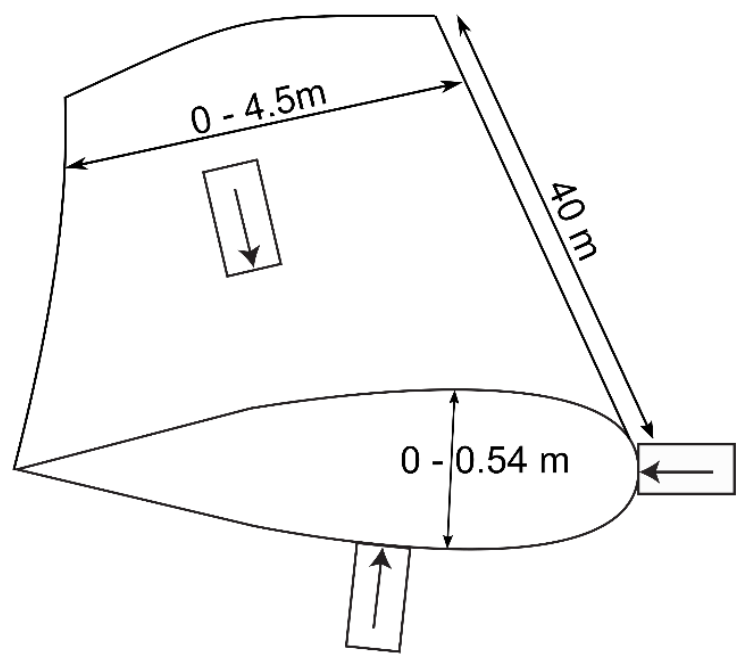

Figure 2: Different clamping orientations required to machine a wind turbine blade, based on the design requirements from (van Houten et al. 2020). Indicative size measurements from (Wang et al. 2015) for a 2MW turbine based on a NACA 63-412 airfoil. The chord and thickness of the blade vary over the length of the blade due to aerodynamic considerations.

\section{Applied search methods}

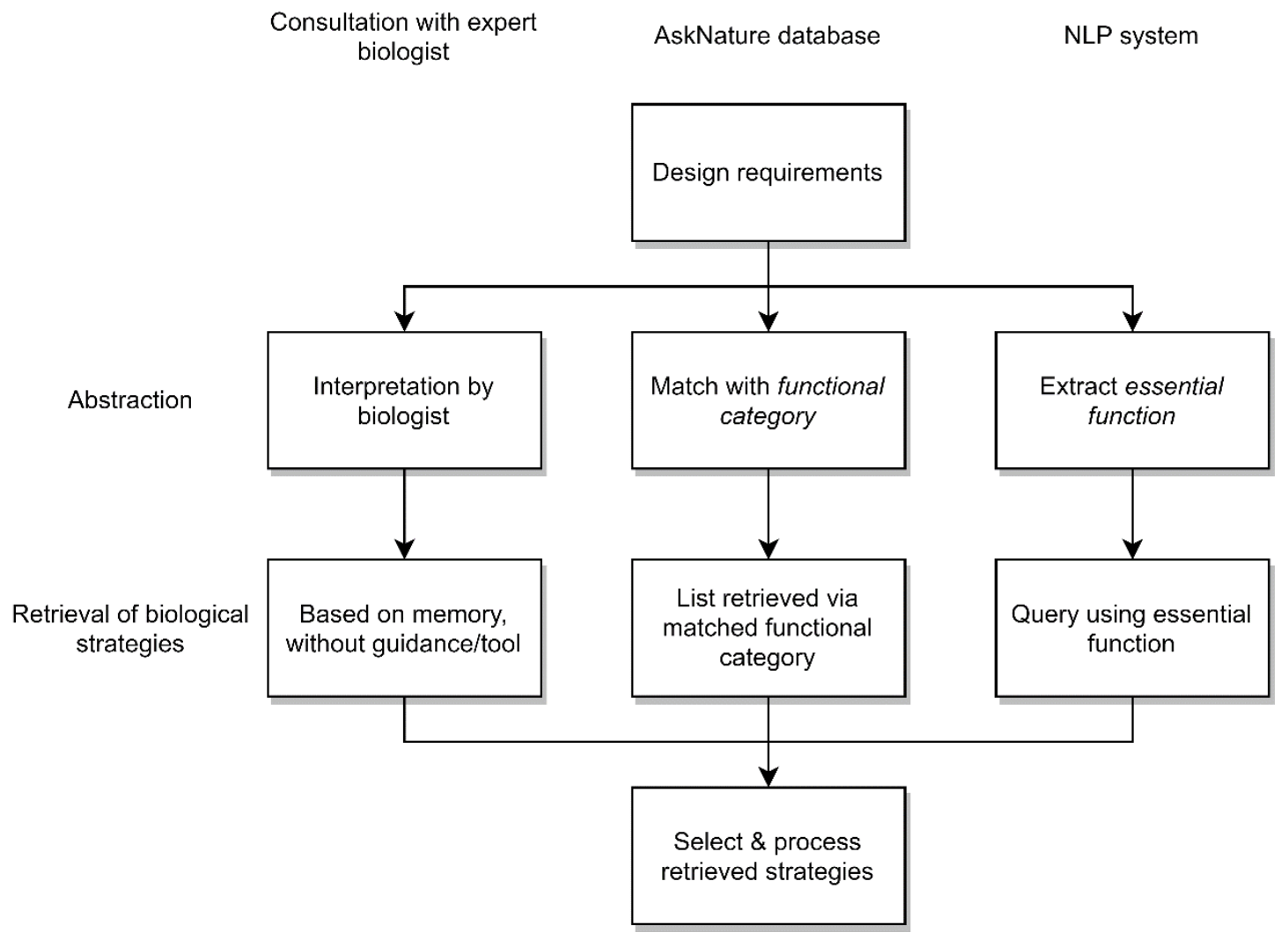

Figure 3: Overview of the different stages in the applied search methods. 
For each of the three previously described approaches, this section details the applied methods to gather bio-inspiration. On a high level, each of the methods has an abstraction and a retrieval stage. During the abstraction stage, the design requirements are processed into a specific form that can be used in the retrieval phase to identify biological strategies. Figure 3 depicts this high-level overview for the applied methods, whilst the remainder of this section details the concrete steps taken.

For the consultation with an expert biologist, a researcher with research interests in insect locomotion and insect systems as a source of bio-inspiration for engineering, received the design requirements. No systematic search method was imposed on the biologist, resulting in a consultation of a biologist as could be organised by a design office enquiring for bio-inspiration. Exhaustiveness was not required for the search. The biologist opted for a memory search, relying on his general knowledge along with some non-systematic keyword searches.

The AskNature database orders a set of curated biological strategies in a taxonomy based on functionality. To retrieve relevant strategies from the database, the desired functionality is matched with the AskNature taxonomy and leads to a function category (Deldin et al. 2014). The design specifications for the case study lead to the function category "attach temporarily". This category contains a set of possibly relevant biological strategies. The design requirements detail a method of attaching to a relatively much larger surface, without control over nor damaging it. Based on these design requirements, a mechanical engineer without a biology background read the description of the retrieved strategies and removed those requiring changes in surface, damaging of the surfaces, dealing with objects of the same relative size or fluids and microbiology.

An elaboration of the NLP system developed in (Vandevenne et al. 2015) was also used to search for biological inspiration. Instead of using product aspects or existing products as a search query, the adapted method uses the essential function that the design will have to fulfil as input. The essential function is defined by Pahl et al. as "the generalized crux of the problem, in a formulation that does not imply any solution principle" (Pahl et al. 2007). To arrive at the required essential function, Pahl et al. propose to use a step-by-step approach to systematically broaden the problem formulation, removing any preconceived solutions. The goal of the design is to link the mobile machining unit and the much larger workpiece with different surface conditions. Generalising this statement and removing the preconceived solutions from the formulation reveals the essential function of "reversibly attaching to a much larger surface". The NLP system returns a ranked list of biological publications in order of relevance. The same mechanical engineer that filtered the AskNature strategies skimmed the titles and abstracts of these publications to determine if they might contain a relevant principle. This is a subjective and error-prone step as will become clear later on. The potentially relevant publications are read in more detail to identify the underlying principle.

Vandevenne et al. (2015) tested their NLP system with a limited corpus consisting of approximately 8000 documents from the Journal of Experimental Biology. More recent text mining policies allow compiling an extensive corpus of biological publications for text mining. The corpus used by this research consists of articles from the journals located in the life sciences section of Science Direct and those found using a keyword search for nature, biology and ecology in the CrossRef repository. These journals were filtered to remove those reporting on human life sciences, agriculture and microbiology, as well as those that do not report in English. Articles are only included in the corpus if they are at least 2000 words long and contain a mention of an organism. The resulting corpus contains 160000 articles of 
which $14 \%$ are open access. The remainder consists of subscription-based content that is accessed using the open text mining policies.

\section{Retrieved inspiration}

During bio-inspired design, the engineer is not trying to directly copy the strategy from nature, but rather aims to extract the underlying principle which delivers the desired function so it can be analogically transferred to the engineering domain (Nagel et al. 2018). Many different organisms might employ the same principle in a strategy adapted to their local living conditions. Therefore, the variety in different working principles is important, not the variety in different organisms per se. In order to compare the different search methods, the strategies were grouped per working principle. Table 1 presents the principles that were found by the different search methods. The next paragraph, along with figure 4 , briefly describes the working principles.

Table 1: The different principles found by each of the search methods: a consultation with an expert biologist, the AskNature database and a NLP system. The symbol * indicates principles that were retroactively identified.

\begin{tabular}{lllll}
\hline & & $\begin{array}{l}\text { Consultation with an } \\
\text { expert biologist }\end{array}$ & Ask Nature & NLP system \\
\hline Adhesive pads & Wet & $\mathrm{X}$ & $\mathrm{X}$ & $\mathrm{X}$ \\
\hline & Dry & $\mathrm{X}$ & $\mathrm{X}$ & $\mathrm{X}$ \\
\hline Mechanical & & $\mathrm{X}$ & $\mathrm{X}$ \\
\hline Suction & & $\mathrm{X}$ & $\mathrm{X}$ \\
\hline Polymers & Threads & & $\mathrm{X}$ & $\mathrm{X}$ \\
\hline \multicolumn{1}{l}{ Capillary } & Mucus & & $\mathrm{X}$ & $\mathrm{X}^{\star}$ \\
\hline
\end{tabular}

Adhesive pads consist of surfaces densely covered in setae, hair like structures that allow the organism to make use of the Vanderwaals force between molecules to attach to a smooth surface (Autumn et al. 2006). The fine points of the setae maximise the contact area. In nature, two different strategies are employed to ensure this contact exists on a rougher surface: a dry system with very fine endings or a wet system with more crude setae that require the use of an adhesive fluid to ensure the contact (Federle 2006). Another principle used in nature for temporary adhesion is suction. For example, Remora attach to their hosts using a suction cup-like device, lowering their metabolic demands for swimming and breathing (Beckert et al. 2015). Mechanical means are also used to keep the relative position of an organism. This principle can be embodied using a claw (Al Bitar et al. 2010) or a pliant skin that hooks behind any surface roughness, generating the friction necessary to prevent relative movement (Gorb et al. 2001). Polymers are also employed to perform adhesion: threads are woven and adhere to the surface (Moeser et al. 2006) or a mucus containing sticky polymers is excreted. The viscoelastic properties of this mucus allow it to be an adhesive and a lubricant at the same time (Lai et al. 2010). Finally, mucus can also create a liquid bridge between two surfaces, causing capillary forces (Grenon et al. 1981). Figure 4 provides a schematic representation of these principles.

An interesting observation made after compiling the list of strategies is that biological systems often apply two principles in tandem, strengthening the effect or covering the weaknesses of one strategy with the other. For example, the suction cup of the Remora also contains small protrusions that are pressed to the rough host surface to generate extra friction in the longitudinal direction, allowing 
Remoras to remain attached to a fast moving host. Certain hexapods (Gorb et al. 2001) and spiders (Kesel 2003) combine mechanical claws with adhesive pads to hold on to smooth and rough surfaces. Tree frog's wet adhesive pads also lean on capillary action to increase their stickiness (Langowski et al. 2018).

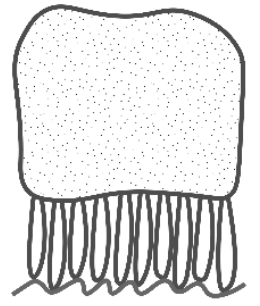

a

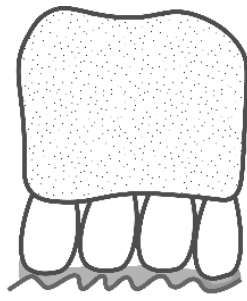

b

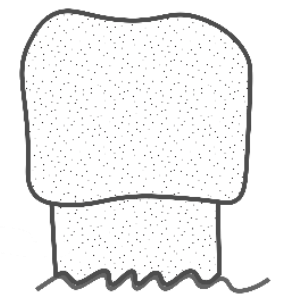

C

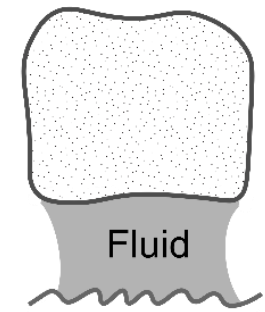

d

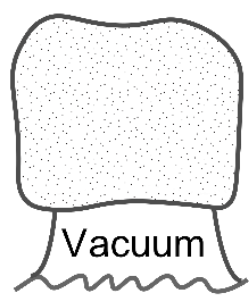

e

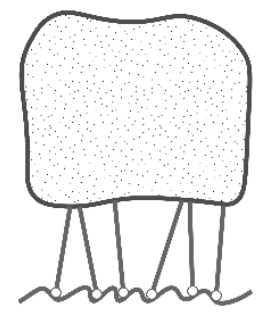

f

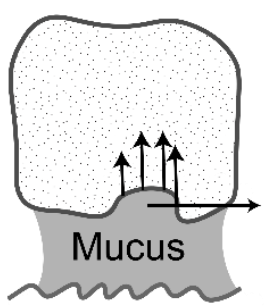

g

Figure 4: Schematic representation of the different identified working principles: (a) dry adhesive pads, (b) wet adhesive pads, (c) mechanical friction with a soft pad, (d) capillary action, (e) suction, (f) polymer threads and (g) polymer mucus. Drawing based on (Federle 2006; Federle et al. 2004; Langowski et al. 2018; Gorb et al. 2001; Peyer et al. 2009).

The biologist readily identified the different adhesive pads used by different sizes of insects and the suction based strategy of the clingfish. No iterations on the bio-inspiration and the problem formulation were made. After looking over the results from the NLP method, the biologist noted that he also had previous experience with the attachment method employed by Lampreys.

In the AskNature database, 91 strategies were available for the chosen taxonomic classification. The mechanical engineer discarded 39 of these strategies based on the previously mentioned criteria and found that five contained duplicate strategies, resulting in 47 strategies relevant to the case study. Identifying the underlying principles resulted in the principles presented in table 1.

A principle is often present in multiple publications, resulting in a redundant list of publications as output from the NLP method. Table 2 presents a selection of the biological articles and their identified principle(s), omitting publications that are describing already present principles or that contain strategies not relevant to the query. The comparison with the principles identified from AskNature revealed that the engineer did not identify the application of mucus and capillary adhesion as relevant principles from the retrieved articles. A reverse search for these principles in the already retrieved biological articles revealed that they were present in the results of the first search query. The failure of the engineer to identify these principles in the first pass is discussed below. The principles found using this reverse validation are marked with a star in table 1. 
Table 2: A selection of biological articles returned by the NLP search system, annotated with the identified principle or reason for rejection.

\begin{tabular}{|c|c|c|c|}
\hline Rank & $\begin{array}{l}\text { Relevance } \\
\text { score }\end{array}$ & Title & Principle \\
\hline 1 & 1.000 & $\begin{array}{l}\text { Muscular architecture of Milnesium tardigradum and } \\
\text { Hypsibius sp. (...) }\end{array}$ & $\begin{array}{l}\text { Not relevant: similar } \\
\text { size }\end{array}$ \\
\hline 2 & 0.899 & $\begin{array}{l}\text { Adhesion of ungerminated conidia of Colletotrichum } \\
\text { graminicola to artificial hydrophobic surfaces }\end{array}$ & Polymer \\
\hline 3 & 0.894 & $\begin{array}{l}\text { Echinostoma caproni in mice: Studies on the } \\
\text { attachment site of an intestinal trematode }\end{array}$ & Suction \\
\hline 12 & 0.676 & $\begin{array}{l}\text { Activity-independent cell adhesion to tissue-type } \\
\text { transglutaminase is mediated by } \alpha 4 \beta 1 \text { integrin }\end{array}$ & $\begin{array}{l}\text { Not relevant: } \\
\text { Microbiology }\end{array}$ \\
\hline 22 & 0.545 & $\begin{array}{l}\text { Attachments by parasitic lampreys within the branchial } \\
\text { cavities of their hosts }\end{array}$ & Suction \\
\hline 31 & 0.518 & Attachment ability of sawfly larvae to smooth surfaces & $\begin{array}{l}\text { Wet adhesion pad \& } \\
\text { mechanical }\end{array}$ \\
\hline 32 & 0.516 & $\begin{array}{l}\text { Changes in materials properties explain the effects of } \\
\text { humidity on gecko adhesion }\end{array}$ & Dry adhesive pads \\
\hline 41 & 0.456 & $\begin{array}{l}\text { Zebra mussels anchor byssal threads faster and tighter } \\
\text { than quagga mussels in flow }\end{array}$ & Polymer \\
\hline 46 & 0.435 & Evolution of locomotory attachment pads of hexapods & $\begin{array}{l}\text { Adhesive pads \& } \\
\text { mechanical }\end{array}$ \\
\hline 54 & 0.416 & Why are so many adhesive pads hairy? & $\begin{array}{l}\text { Wet \& dry adhesive } \\
\text { pads }\end{array}$ \\
\hline 62 & 0.392 & $\begin{array}{l}\text { Tarsal morphology and attachment ability of the codling } \\
\text { moth Cydia pomonella L. (Lepidoptera, Tortricidae) to } \\
\text { smooth surfaces }\end{array}$ & $\begin{array}{l}\text { Wet adhesion pad \& } \\
\text { claw }\end{array}$ \\
\hline 105 & 0.317 & $\begin{array}{l}\text { Tree frog attachment: mechanisms, challenges, and } \\
\text { perspectives }\end{array}$ & $\begin{array}{l}\text { Capillary \& wet } \\
\text { adhesive pads }\end{array}$ \\
\hline 188 & 0.250 & $\begin{array}{l}\text { Remora fish suction pad attachment is enhanced by } \\
\text { spinule friction }\end{array}$ & Suction \& mechanical \\
\hline 192 & 0.248 & $\begin{array}{l}\text { The tenacity of the limpet, Patella vulgata L.: An } \\
\text { experimental approach }\end{array}$ & Polymer mucus \\
\hline
\end{tabular}

\section{Discussion}

The expert biologist performed the information gathering process without a prescribed search methodology nor the target of creating an exhaustive list of bio-inspiration. Relying on previous knowledge and some keyword based searches, the biologist identified the strategies located in his area of expertise. However, biologists tend to specialise and cannot be expected to instantly come up with exhaustive knowledge of a broad range of strategies. In contrast, combining different principles in one strategy is a meta-strategy applied in some biological systems. While the target is never to provide an exhaustive list of relevant biological strategies, providing a representative sample of the whole area of biology might increase the effectivity of the generated solution and requires the consulted biology expert to be either broadly trained or use a systematic search method in literature. 
Including a biologist in the entire design process mitigates the risk posed in the previous paragraph by allowing for multiple iterations of the bio-inspiration. This also has the advantage of allowing the biology expert to assist in the transfer of the biological knowledge to the engineering solution.

Of the 91 strategies in the AskNature taxonomy branch for temporary adhesion, only approximately half are relevant to the current case study. In these 47 strategies, all of the identified principles applicable to the case study are present. However, this branch of the taxonomy is among the few more densely populated categories. In contrast, a large number of function level categories are nearly empty. Figure 5 displays the number of strategies that are present in the different function level categories, indicating the temporary attachment category with a diamond. Despite many years of effort, not all categories of the taxonomy contain a substantial number of strategies. Whilst a well filled category returns a good number of principles and is as a representative source of bio-inspiration, the large amount of effort required to complete all of the categories to a satisfiable level stops this method from being scalable to cover the whole biology domain. A more scalable method is clearly desirable. This is one of the more filled functional categories in the taxonomy, offering a chance to compare the recall performance of the other methods.

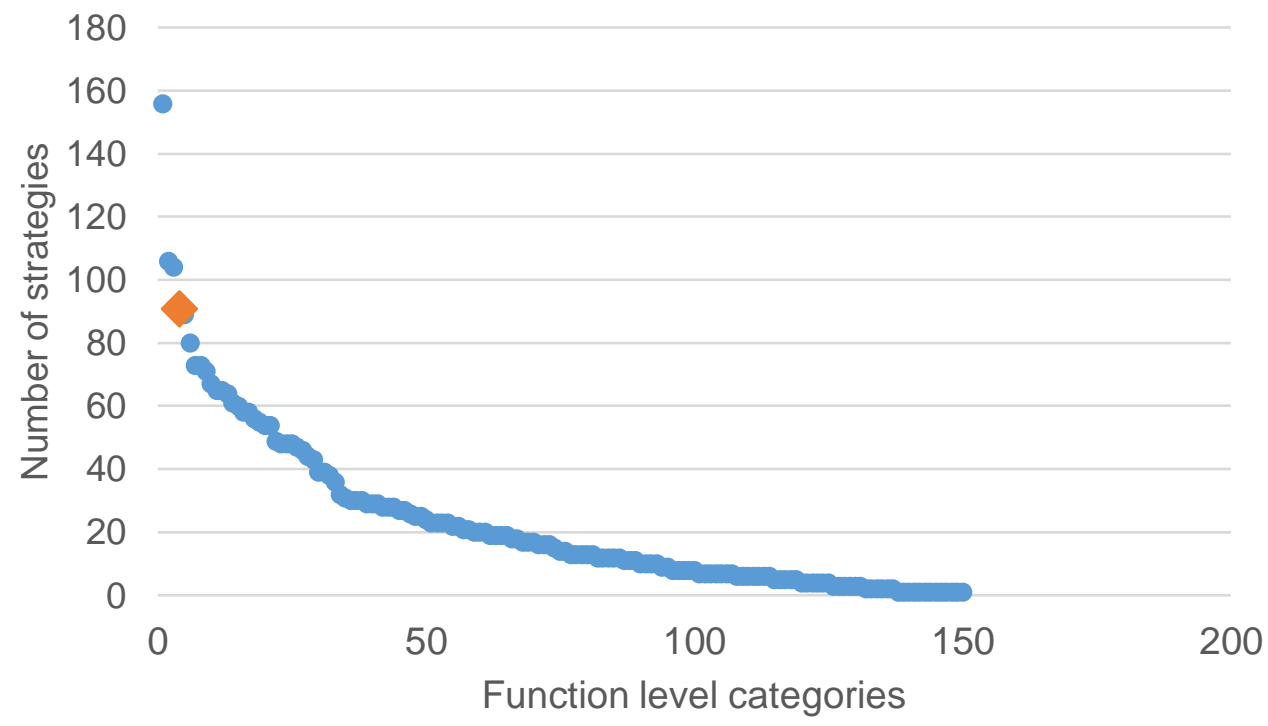

Figure 5: The number of strategies that are present in the different function level categories. The diamond indicates the temporary adhesion function category. Data from the AskNature website (January 29 2020).

The NLP method managed to supply relevant biological articles that contain at least the relevant principles that are present in a well-stocked category of AskNature. However, reading, understanding and extracting the principles from the publications is a time-consuming task and caused the engineer to miss relevant principles. The large number of articles to process limits the possible depth of the analysis. Whilst the method allows engineers to search in biological literature, it currently does not aid them in analysing, selecting and transferring the relevant strategies to the engineering domain. Here the inclusion of an expert biologist in an interdisciplinary design team offers the opportunity to reduce the time required to understand, filter and transfer the principles found in the biological publications. 
Future research making the systematic search method viable in a design environment is necessary. The development of an automatic method of grouping the biological publications per biological strategy or underlying principle is desirable. This grouping aids the engineer or biologist using the search method in quickly assessing the relevance of a number of publications, supporting the next steps in the bio-inspired design process.

The presented study highlighted the potential of the NLP methodology to find a more representative set of biological principles in a scalable way using biological literature. However, some limitations should be pointed out. First, only one specific case is provided for the comparison. Furthermore, only one round of collecting bio-inspiration was performed: the problem formulation and query are not refined based on the analysis of the found biological analogies, preventing the biologist from influencing the result and being integrated in the design team. Finally, the comparison of the different methods is based on the identified working principles, while research has shown that engineers can extract different analogies based on the principle, but also based on the complete system and even form, of one biological system (Nagel et al. 2018).

\section{Conclusion}

Three different methods for identifying bio-inspiration were compared based on their recall performance. It is found that the NLP system that draws on the combined knowledge of many biologists automatically identified articles containing description of all relevant working principles found in the AskNature database and identified by a single biologist during a consultation akin to a design bureau inquiring for biological inspiration. This indicates the potential of NLP systems for systematic search strategies for biological inspiration. As a limitation, it should be noted that this is based on the results for only a single design.

Processing the retrieved biological articles is still a difficult and time-consuming task where the support of a biologist could be beneficial. Furthermore, the inclusion of a biologist in the design process could save time and lead to more effective biological analogies due to the continuous evaluation of the biological strategies. In order to make the NLP method viable in a design environment, further improvements should help the engineer identify the relevant biological strategies and then support the analogical transfer to the engineering domain.

\section{Acknowledgements}

This work was supported by the Fraunhofer-Gesellschaft Think Tank project BioMANU II, the KU Leuven Research Fund trough project GOA/15/012-SUMMA and the Israel Science Foundation trough grant 2306/18.

\section{References}

Ahmed-Kristensen, Saeema, Bo T. Christensen, and Torben A. Lenau. 2014. "Naturally Original: Stimulating Creative Design through Biological Analogies and Random Images." In DS77 : Proceedings of the DESIGN 2014 13th International Design Conference, 13:427-36. Design. Dubrovnik.

Al Bitar, Loris, Dagmar Voigt, Claus P.W. Zebitz, and Stanislav N. Gorb. 2010. "Attachment Ability of the Codling Moth Cydia Pomonella L. to Rough Substrates." Journal of Insect Physiology 56 (12): 1966-72. https://doi.org/10.1016/j.jinsphys.2010.08.021. 
Autumn, K., A. Dittmore, D Santos, M. Spenko, and M. Cutkosky. 2006. "Frictional Adhesion: A New Angle on Gecko Attachment." Journal of Experimental Biology 209 (18): 3569-79. https://doi.org/10.1242/jeb.02486.

Beckert, Michael, Brooke E. Flammang, and Jason H. Nadler. 2015. "Remora Fish Suction Pad Attachment Is Enhanced by Spinule Friction." Journal of Experimental Biology 218 (22): 3551-58. https://doi.org/10.1242/jeb.123893.

Biomimicry Institute. 2020. "AskNature - Innovation Inspired by Nature." January 17, 2020. https://asknature.org/.

Byrne, Gerald, Dimitri Dimitrov, Laszlo Monostori, Roberto Teti, Fred van Houten, and Rafi Wertheim. 2018. "Biologicalisation: Biological Transformation in Manufacturing." CIRP Journal of Manufacturing Science and Technology 21 (May): 1-32. https://doi.org/10.1016/j.cirpj.2018.03.003.

Deldin, Jon-Michael, and Megan Schuknecht. 2014. "The AskNature Database: Enabling Solutions in Biomimetic Design." In Biologically Inspired Design: Computational Methods and Tools, edited by Ashok K Goel, Daniel A McAdams, and Robert B. Stone, 17-27. London: Springer London. https://doi.org/10.1007/978-1-4471-5248-4_2.

Fayemi, Pierre-Emmanuel, Kristina Wanieck, Cordt Zollfrank, Nicolas Maranzana, and Améziane Aoussat. 2017. "Biomimetics: Process, Tools and Practice." Bioinspiration \& Biomimetics 12 (1): 011002. https://doi.org/10.1088/1748-3190/12/1/011002.

Federle, Walter. 2006. "Why Are so Many Adhesive Pads Hairy?" Journal of Experimental Biology 209 (14): 2611-21. https://doi.org/10.1242/jeb.02323.

Federle, Walter, Werner Baumgartner, and Bert Hölldobler. 2004. "Biomechanics of Ant Adhesive Pads: Frictional Forces Are Rate- and Temperature-Dependent." Journal of Experimental Biology 207 (1): 67-74. https://doi.org/10.1242/jeb.00716.

Gorb, S., and R. Beutel. 2001. "Evolution of Locomotory Attachment Pads of Hexapods." Naturwissenschaften 88 (12): 530-34. https://doi.org/10.1007/s00114-001-0274-y.

Graeff, Eliot, Nicolas Maranzana, and Améziane Aoussat. 2019. "Engineers' and Biologists' Roles during Biomimetic Design Processes, Towards a Methodological Symbiosis." In Proceedings of the Design Society: International Conference on Engineering Design, 1:319-28. https://doi.org/10.1017/dsi.2019.35.

Grenon, J.-F., and G. Walker. 1981. "The Tenacity of the Limpet, Patella Vulgata L.: An Experimental Approach." Journal of Experimental Marine Biology and Ecology 54 (3): 277-308. https://doi.org/10.1016/0022-0981(81)90162-3.

Hashemi Farzaneh, Helena, Katharina Helms, and Udo Lindemann. 2015. "Visual Representations as a Bridge for Engineers and Biologists in Bio-Inspired Design Collaborations." In ICED15, 215-24. ICED. Milan, Italy: Design Society.

Hashemi Farzaneh, Helena, and Udo Lindemann. 2019. A Practical Guide to Bio-Inspired Design. Berlin, Heidelberg: Springer Berlin Heidelberg. https://doi.org/10.1007/978-3-662-57684-7.

Helms, Michael, Swaroop S. Vattam, and Ashok K. Goel. 2009. "Biologically Inspired Design: Process and Products." Design Studies 30 (5): 606-22. https://doi.org/10.1016/j.destud.2009.04.003.

Helten, Katharina, Sebastian Schenkl, and Udo Lindemann. 2011. "Biologizing Product Development Results from a Student Project." In Proceedings of the International Conference on Research into Design, 27-34. Bangalore, India.

Houten, Fred van, Rafael Wertheim, Amir Ayali, Elena Poverenov, Guy Mechraz, Udo Eckert, Hendrik Rentzsch, Ines Dani, Mart Willocx, and Joost Duflou. 2020. "Bio-Based Design Methodologies for Products, Processes, Machine Tools and Production Systems." In Press. 
Kesel, A. B. 2003. "Adhesion Measurements on the Attachment Devices of the Jumping Spider Evarcha Arcuata." Journal of Experimental Biology 206 (16): 2733-38. https://doi.org/10.1242/jeb.00478.

Keshwani, Sonal, Torben Anker Lenau, Saeema Ahmed-Kristensen, and Amaresh Chakrabarti. 2017. "Comparing Novelty of Designs from Biological-Inspiration with Those from Brainstorming." Journal of Engineering Design 28 (10-12): 654-80. https://doi.org/10.1080/09544828.2017.1393504.

Kruiper, Ruben, Julian F. V. Vincent, Eitan Abraham, Rupert C. Soar, loannis Konstas, Jessica ChenBurger, and Marc P. Y. Desmulliez. 2018. "Towards a Design Process for Computer-Aided Biomimetics." Biomimetics 3 (3): 14. https://doi.org/10.3390/biomimetics3030014.

Lai, Janice H., Juan C. del Alamo, Javier Rodríguez-Rodríguez, and Juan C. Lasheras. 2010. "The Mechanics of the Adhesive Locomotion of Terrestrial Gastropods." Journal of Experimental Biology 213 (22): 3920-33. https://doi.org/10.1242/jeb.046706.

Langowski, Julian K. A., Dimitra Dodou, Marleen Kamperman, and Johan L. van Leeuwen. 2018. "Tree Frog Attachment: Mechanisms, Challenges, and Perspectives." Frontiers in Zoology 15 (1): 32. https://doi.org/10.1186/s12983-018-0273-x.

Lenau, Torben, Katharina Helten, Clemens Hepperle, Sebastian Schenkl, and Udo Lindemann. 2011. "Reducing Consequences of Car Collision Using Inspiration from Nature." In Proceedings of IASDR2011 : The 4th World Conference on Design Research, 10. Delft, Netherlands: TU Delft.

Lenau, Torben, Anna-Luise Metze, and Thomas Hesselberg. 2018. "Paradigms for Biologically Inspired Design." In Bioinspiration, Biomimetics, and Bioreplication VIII, edited by Akhlesh Lakhtakia, 1. Denver, United States: SPIE. https://doi.org/10.1117/12.2296560.

Moeser, Gretchen M., and Emily Carrington. 2006. "Seasonal Variation in Mussel Byssal Thread Mechanics." Journal of Experimental Biology 209 (10): 1996-2003. https://doi.org/10.1242/jeb.02234.

Nagel, Jacquelyn. 2010. "Systematic Design of Biologically-Inspired Engineering Solutions (Doctoral Dissertation)." https://ir.library.oregonstate.edu/concern/graduate_thesis_or_dissertations/z603r138q.

Nagel, Jacquelyn, Linda Schmidt, and Werner Born. 2018. "Establishing Analogy Categories for BioInspired Design." Designs 2 (4): 47. https://doi.org/10.3390/designs2040047.

Pahl, G., Ken Wallace, Luciënne Blessing, and G. Pahl, eds. 2007. Engineering Design: A Systematic Approach. 3rd ed. London: Springer. https://doi.org/10.1007/978-1-84628-319-2.

Peyer, S. M., A. J. McCarthy, and C. E. Lee. 2009. "Zebra Mussels Anchor Byssal Threads Faster and Tighter than Quagga Mussels in Flow." Journal of Experimental Biology 212 (13): 2027-36. https://doi.org/10.1242/jeb.028688.

Schöfer, Malte, Nicolas Maranzana, Améziane Aoussat, Giacomo Bersano, and Stéphanie Buisine. 2018. "Distinct and Combined Effects of Disciplinary Composition and Methodological Support on Problem Solving in Groups." Creativity and Innovation Management 27 (1): 102-15. https://doi.org/10.1111/caim.12258.

Shu, L.H. 2010. "A Natural-Language Approach to Biomimetic Design." Artificial Intelligence for Engineering Design, Analysis and Manufacturing 24 (04): 507-19. https://doi.org/10.1017/S0890060410000363.

Shu, L.H., K. Ueda, I. Chiu, and H. Cheong. 2011. "Biologically Inspired Design." CIRP Annals 60 (2): 67393. https://doi.org/10.1016/j.cirp.2011.06.001.

Vandevenne, Dennis, Thomas Pieters, and Joost R. Duflou. 2016. "Enhancing Novelty with KnowledgeBased Support for Biologically-Inspired Design." Design Studies 46 (September): 152-73. https://doi.org/10.1016/j.destud.2016.05.003. 
Vandevenne, Dennis, Paul-Armand Verhaegen, Simon Dewulf, and Joost R. Duflou. 2015. "SEABIRD:

Scalable Search for Systematic Biologically Inspired Design." Artificial Intelligence for Engineering Design, Analysis and Manufacturing 30 (01): 78-95. https://doi.org/10.1017/S0890060415000177.

Vincent, Julian F.V, Olga A Bogatyreva, Nikolaj R Bogatyrev, Adrian Bowyer, and Anja-Karina Pahl. 2006. "Biomimetics: Its Practice and Theory." Journal of The Royal Society Interface 3 (9): 471-82. https://doi.org/10.1098/rsif.2006.0127.

Wang, Qiong, Kexiang Wei, Xuejun Li, and Xiaofeng Geng. 2015. "Design and Analysis of Multi-Megawatt Wind Turbines Blades," Journal of Vibroengineering, 17 (7): 10.

Wanieck, Kristina, Pierre-Emmanuel Fayemi, Nicolas Maranzana, Cordt Zollfrank, and Shoshanah Jacobs. 2017. "Biomimetics and Its Tools." Bioinspired, Biomimetic and Nanobiomaterials 6 (2): 53-66. https://doi.org/10.1680/jbibn.16.00010. 